\title{
High performance electrochemical and electrothermal artificial muscles from twist-spun carbon nanotube yarn
}

\author{
Jae Ah Lee ${ }^{1}$, Ray H Baughman ${ }^{2}$ and Seon Jeong Kim ${ }^{1 *}$
}

\begin{abstract}
High performance torsional and tensile artificial muscles are described, which utilize thermally- or electrochemicallyinduced volume changes of twist-spun, guest-filled, carbon nanotube (CNT) yarns. These yarns were prepared by incorporating twist in carbon nanotube sheets drawn from spinnable CNT forests. Inserting high twist into the CNT yarn results in yarn coiling, which can dramatically amplify tensile stroke and work capabilities compared with that for the non-coiled twisted yarn. When electrochemically driven in a liquid electrolyte, these artificial muscles can generate a torsional rotation per muscle length that is over 1000 times higher than for previously reported torsional muscles. All-solid-state torsional electrochemical yarn muscles have provided a large torsional muscle stroke $\left(53^{\circ}\right.$ per $\mathrm{mm}$ of yarn length) and a tensile stroke of up to $1.3 \%$ when lifting loads that are 25 times heavier than can be lifted by the same diameter human skeletal muscle. Over a million torsional and tensile actuation cycles have been demonstrated for thermally powered CNT hybrid yarns muscles filled with paraffin wax, wherein a muscle spins a rotor at an average 11,500 revolutions/minute or delivers $3 \%$ tensile contraction at 1200 cycles/minute. At lower actuation rates, these thermally powered muscles provide tensile strokes of over $10 \%$.
\end{abstract}

Keywords: Artificial muscle; Yarn muscle; Carbon nanotube muscle; Electrochemical actuator; Thermal actuator

\section{Introduction}

Carbon nanotube (CNT) sheets drawn from spinnable CNT forests has been investigated for such applications as actuators [1-3], supercapacitors [4,5], solar cells [6], biofuel cells [7], and acoustic speaker [8]. There are many other potential application areas because of such properties as high electrical conductivity, high mechanical properties, optical transparency for single sheets, and high gravimetric surface area $[4,9]$. Zhang et al. developed methods to produce these sheets from spinnable CNT forest [9] and Lima et al. exploited these sheets for their biscrolling process, in which guest-coated CNT sheets are spun into yarn in which the guest is trapped in helical yarn corridors [10]. These hybrid yarns can contain up to $99 \mathrm{wt} \%$ of guest and still remain flexible and retain the original properties of the CNT host and guest materials.

\footnotetext{
* Correspondence: sjk@hanyang.ac.kr

${ }^{1}$ Center for Bio-Artificial Muscle and Department of Biomedical Engineering, Hanyang University, Seoul 133-791, South Korea

Full list of author information is available at the end of the article
}

CNT tensile actuators and cantilever-based bending actuators, powered by electricity, fuels, light, or heat, have been developed during the past few decades [11]. While electrostatically driven actuation of forest-drawn CNT sheets can generate giant stokes (220\%) and giant elongation rates $\left(3.7 \times 10^{4} \% / \mathrm{s}\right)$, these ultralight aerogelbased actuators require high voltages and are not scalable in thickness to generate high forces [12]. Electrochemical actuation (by double-layer charge injection) of CNT sheets fabricated by solution processing provides very small strokes $(\sim 0.2 \%)$ [13]. Torsional and rotational motors that use single nanotubes have been demonstrated [14], but the nanotubes do not produce the actuation.

In this study, we describe methods for producing high performance CNT-based torsional and tensile artificial muscles by processes that involve inserting twist into forest-drawn CNT sheets. All of these muscles use yarn volume expansion (produced either electrochemically or by thermal expansion of a yarn guest) to reversibly generate yarn contraction and yarn untwist [1,2]. Yarn coiling, produced by inserting high twist into the CNT yarn, 
dramatically amplifies tensile stroke and work capabilities compared with those for non-coiled yarn [2].

\section{Review}

\subsection{Electrochemically driven liquid-state and all-solid- state CNT yarn muscles}

The precursor yarns used for electrochemical muscles $[1,3]$ were similarly made as those described for thermally powered hybrid muscles [2]. These yarns were fabricated by drawing CNT sheets from a CNT forest (Figure 1a) and inserting twist into a sheet or sheet stack to make a twisted CNT yarn. Twist insertion during forest draw is shown in Figure 1b. Figure 1c and d show, respectively, SEM images of a twisted, non-coiled single-ply yarn and a coiled single-ply yarn that has been coiled as a result of very high twist insertion. The utilized 300$400 \mu \mathrm{m}$ high CNT forests, from which the CNT sheets were drawn, were prepared by chemical vapor deposition on an iron-catalyst-supporting silicon wafer, using acetylene gas as the carbon precursor. These forests comprised multi-walled CNTs that had an outer diameter of $\sim 10 \mathrm{~nm}$ and contain approximately nine walls.

By applying a few volts potential between a twist-spun, non-coiled CNT yarn and a counter-electrode in an electrolyte bath (Figure 2a), Foroughi et al. [1] demonstrated that yarn artificial muscles can torsionally rotate a relatively heavy paddle to generate a rotation per length that is over 1000 times higher than for previously reported torsional muscles made from a shape-memory alloy [15], piezoelectric ceramics [16], or conducting polymer [17]. Only one-half of the pictured CNT yarn (which is tethered to prevent torsional end displacement) is submerged in the electrolyte (so that this half provides the torsional actuation, and the second half functions as a return spring to enable highly reversible torsional and tensile actuation). The paddle used to measure torsional rotation was located at near yarn center and the cantilever supporting the top end of the muscle enabled measurement of tensile actuation.

Figure $2 \mathrm{~b}$ shows torsional rotation and tensile actuation versus time for a CNT yarn muscle (with a $40^{\circ}$ yarn bias angle for CNT orientation with respect to the yarn axis) that uses the configuration of Figure 2a. The actuating $12 \mu \mathrm{m}$ diameter yarn functioned as a torsional artificial muscle to provide a reversible $15,000^{\circ}$ rotation and a maximum torsional rotation rate of 590 revolutions per minute, while rotating a paddle that was 830 times the diameter of the actuating yarn and 1800 times its mass. To achieve this result the $120 \mathrm{~mm}$ long muscle yarn was pulsed to $+5 \mathrm{~V}$ (versus $\mathrm{Ag} / \mathrm{Ag}+$ reference) and then to $0 \mathrm{~V}$ for about $5 \mathrm{~s}$ each (while using as liquid electrolyte $0.2 \mathrm{M}$ tetrabutylammonium hexafluorophosphate $\left(\mathrm{TBA}^{\mathrm{P}} \mathrm{PF}_{6}\right)$ in acetonitrile). The initial paddle acceleration from the Figure $2 \mathrm{~b}$ results was $\alpha=50 \mathrm{rad} / \mathrm{s}^{2}\left(9000^{\circ} / \mathrm{s}^{2}\right)$, which together with the paddles moment of inertia, provides a maximum starting torque of $1.85 \mathrm{~N} \cdot \mathrm{m}$ per kilogram of actuating yarn mass. This specific torque is similar to that achieved by large commercial electric motors $(2.5$ to $6 \mathrm{~N}$. $\mathrm{m} / \mathrm{kg}$ ). The peak torsional power output (above $61 \mathrm{~W} / \mathrm{kg}$, based on actuating yarn mass) can be compared with the $300 \mathrm{~W} / \mathrm{kg}$ realized by large electric motors. This torsional muscle simultaneously acts as a tensile muscle, thereby providing an additional peak power contribution of $920 \mathrm{~W} / \mathrm{kg}$ by contracting against the applied $88 \mathrm{MPa}$ load, which is equivalent to lifting a mass 185,000 times the mass of the actuating muscle in $1.2 \mathrm{~s}$.
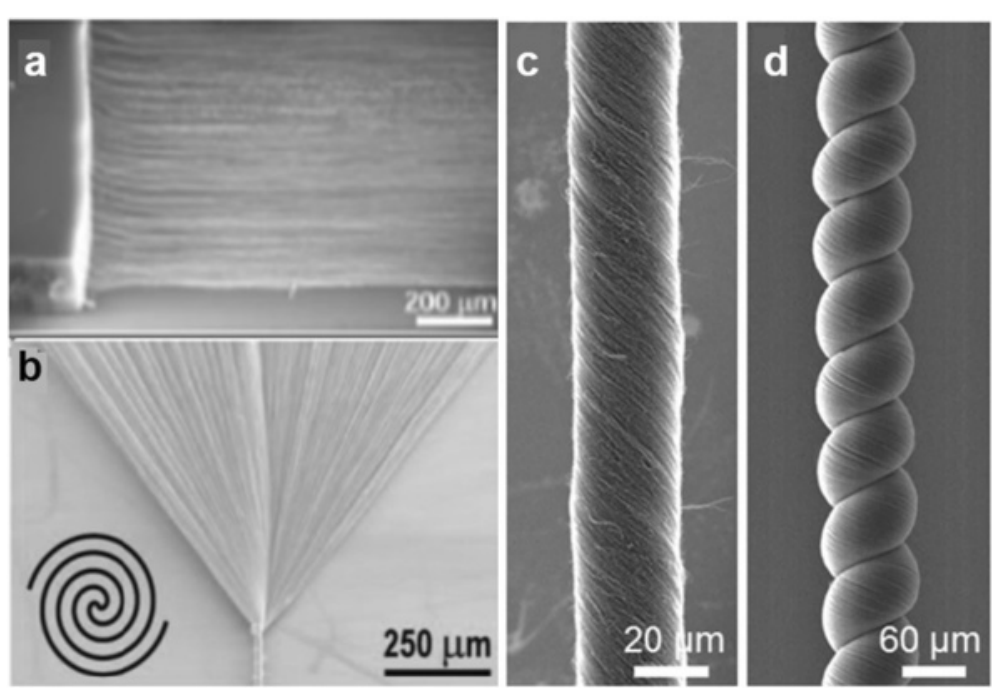

Figure 1 MWNT sheets and yarn structures for torsional and tensile actuation. SEM images of (a) a CNT sheet being drawn from a CNT forest (which is on the left in this image), (b) Fermat-type twist insertion during spinning a yarn from a CNT forest, (c) a single-ply, twisted, non-coiled CNT yarn, and (d) a single-ply, coiled yarn [2]. 


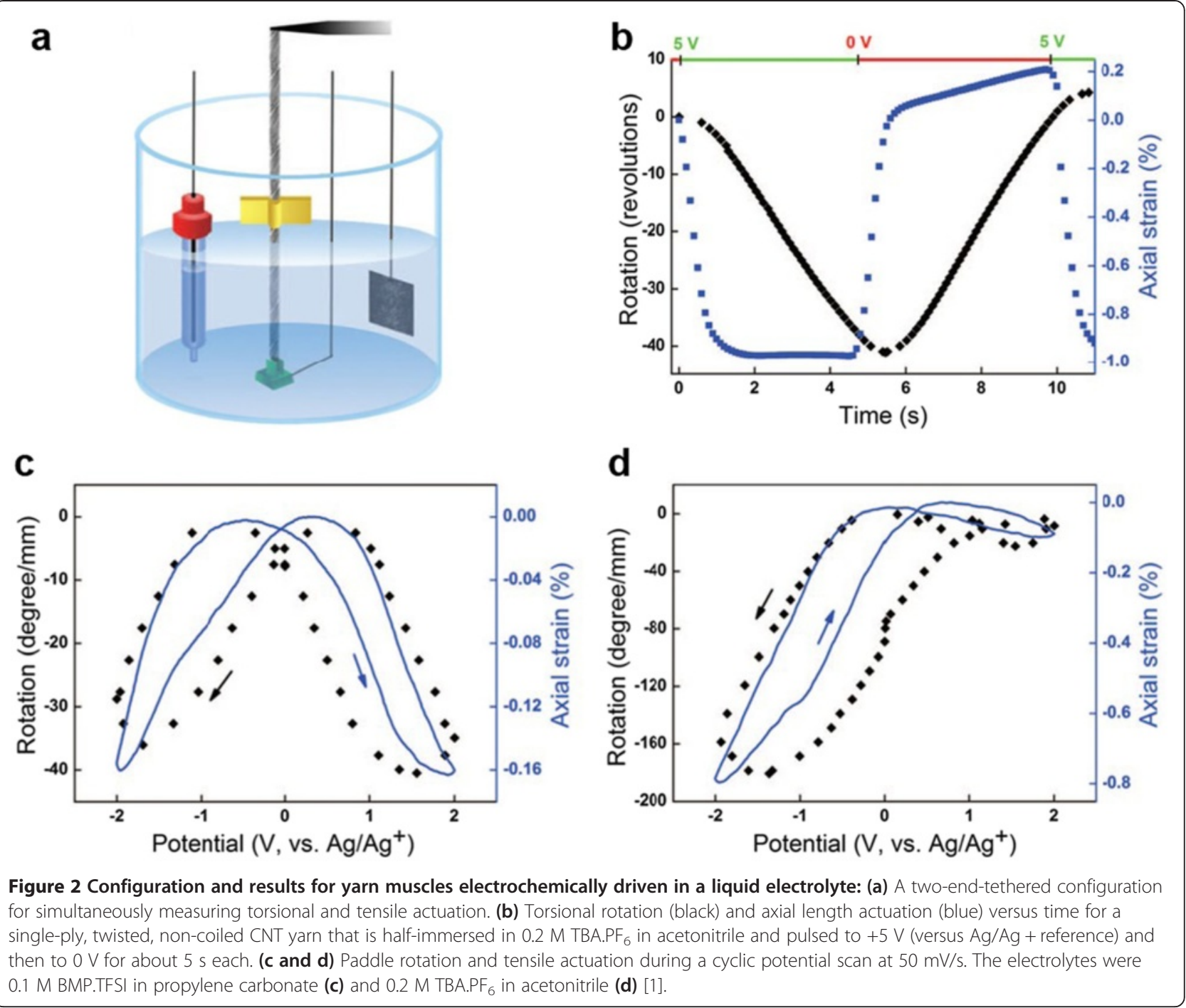

Figure $2 \mathrm{c}$ and $\mathrm{d}$ shows that torsional actuation and yarn tensile actuation are correlated and both depend on the size of the electrolyte ion used to compensate electronic charge. When a 1-butyl-1-methylpyrrolidinium bis(trifluoromethanesulfonyl) imide (BMP.TFSI) electrolyte was used (Figure 2c), which has similar van der Waals volumes [18] of the anion $\left(147 \AA^{3}\right)$ and cation $\left(167 \AA^{3}\right)$, similar torsional and tensile actuation was shown at both positive and negative potentials. Likewise, the much large actuation during reduction than for oxidation in Figure $2 \mathrm{~d}$ for the TBA.PF 6 electrolyte reflects the larger unsolvated van der Waals volume [18] for the TBA cation $\left(293 \AA^{3}\right)$ than for the $\mathrm{PF}_{6}$ anion $\left(69 \AA^{3}\right)$.

While these advances using liquid electrolytes are fundamentally important (and use of the torsional muscles in a microfluidic circuit was demonstrated) [1], more general practical utilization is restricted by the use of a liquid electrolyte bath and the associated need for a containment system, which dramatically degrades the gravimetric and volumetric performance of the overall actuator device.

Lee et al. [3] eliminated these problems by showing that laterally connected anode and cathode CNT yarns could be combined in one torsional yarn muscles (Figure 3), thereby enabling all-solid-state electrochemical muscles that provide giant torsional actuation. Identical twisted, non-coiled anode and cathode yarns were made by inserting twist into forest-drawn CNT sheets, and then infiltrating these yarns with a gel electrolyte (poly(vinylidene fluoride-co-hexafluoropropylene) (PVDF-co-HFP), containing tetraethyl ammonium tetrafluoroborate $\left(\mathrm{TEA} . \mathrm{BF}_{4}\right)$ with propylene carbonate (PC)). These two electrolyte-coated yarns were plied together using opposite twist directions for yarn plying than for the initially introduced yarn twist (Figure 3c, d). This use of opposite twist directions for yarn twist and yarn plying is important, since it results in a torque-balanced system when no 


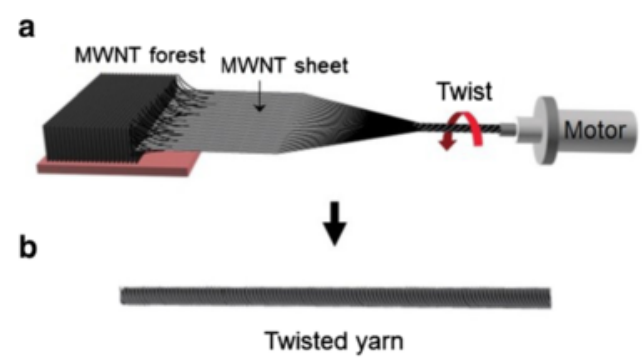

e

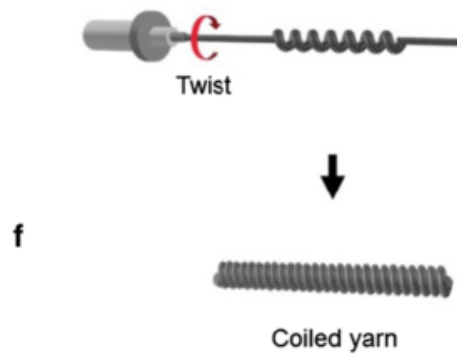

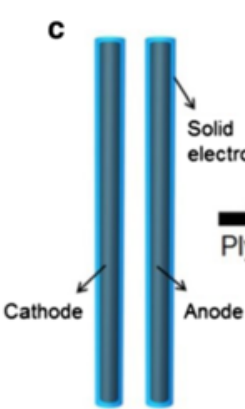

g

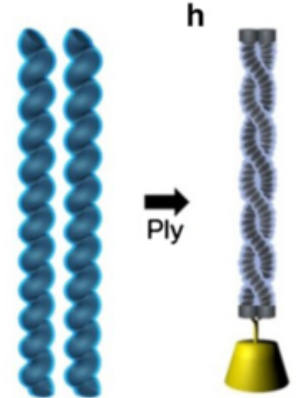

Figure 3 Illustration of yarn fabrication methods for torsional and tensile electrochemical CNT muscles. (a) A CNT sheet was pulled from a CNT forest as twist was inserted to provide yarn for the torsional muscles. Inserting twist into stacks of forest-drawn CNT sheets enabled increases in yarn diameter. (b) A Twisted, non-coiled CNT yarn, containing about 12,000 to 15,000 turns/m of inserted twist when a single CNT sheet was used for yarn fabrication. (c) Anode and cathode yarns infiltrated and coated with PVDF-co-HFP based TEA.BF 4 solid gel electrolyte. (d) Illustration of a plied, non-coiled yarn muscle with attached paddle for torsional actuation measurements, which had been over coated with the above electrolyte after plying two identical electrolyte-filled CNT yarns. (e) Illustration of the insertion of additional twist to produce yarn coiling, which was deployed to provide the fully coiled yarns used for tensile muscles. (f) Fully-coiled yarn used as both muscle anode and cathode for tensile muscles. (g) Coiled anode and cathode yarns that are infiltrated and coated with $\mathrm{PVA}^{-b a s e d ~} \mathrm{H}_{2} \mathrm{SO}_{4}$ solid gel electrolyte. (h) Illustration of a plied, coiled yarn muscle with attached weight for tensile actuation measurements, which had been over coated with the above electrolyte after plying [3].

voltage is applied between anode and cathode yarns, which enables reversible actuation.

Torsional actuation was obtained using the configuration of Figure 3d, where a one-end-tethered, plied, non-coiled yarn supports a paddle on the free end. Fast, reversible torsional strokes $\left(14-53^{\circ} / \mathrm{mm}\right)$ with small hysteresis resulted when using a PVDF-co-HFP/TEA.BF $/$ PC solid gel electrolyte and applied square-wave voltages between 2.5 and $5 \mathrm{~V}$ (Figure $4 \mathrm{a}$ and b). A gravimetric peak torque of $0.067 \mathrm{~N} \cdot \mathrm{m} / \mathrm{kg}(3.05 \mathrm{nN} \cdot \mathrm{m})$ was generated for this $29 \mu \mathrm{m}$ diameter yarn by applying a square-wave voltage of $5 \mathrm{~V}$.

To obtain enhanced tensile actuation for all-solid-state electrochemical muscles, identical anode and cathode yarns in the muscles were coiled (Figure $3 e$ and f). So that we could more conveniently investigate tensile actuation, large diameter CNT yarns were made by inserting twist into a 30-layer stack of forest-drawn sheets. After simultaneously infiltrating and coating both anode and cathode yarn with an aqueous gel electrolyte (polyvinyl alcohol (PVA) in $1 \mathrm{M}$ aqueous sulfuric acid) left-handed coiled electrodes ( $\mathrm{Z}$ twist) were plied by $\mathrm{S}$ twist (righthanded twist) to form a torque-balanced, two-ply, solidstate muscle (Figures $3 g$ and $h$ ).
A reversible $0.52 \%$ tensile contraction was obtained for this electrochemical muscle (in the configuration of Figure $3 \mathrm{~h}$ ) when a square-wave voltage of $1 \mathrm{~V}$ was applied to lift a load that provided an $11 \mathrm{MPa}$ stress (Figure 4c). This load lifting capability is 27 times that of human skeletal muscle. Importantly, this electrochemically driven all-solid-state muscle provided a latched state, where charging-induced contraction was largely maintained after subsequent disconnection from the power supply (Figure 4d). In fact, 91.5\% of the muscle contraction was maintained for one hour following discontinuation of the applied voltage.

\subsection{Thermally and electrothermally powered CNT yarn hybrid muscles}

This last category of twist-spun yarn muscles is powered by temperature changes, which can be produced by such processes as electrical heating (using the electrical conductivity of the CNTs), changes in environmental temperature, absorption of light or other electromagnetic radiation, or chemical reactions. These muscles are called hybrid CNT yarn muscles, since the thermally driven volume expansion of a guest within the twist-spun CNT yarn drives the reversible contraction 

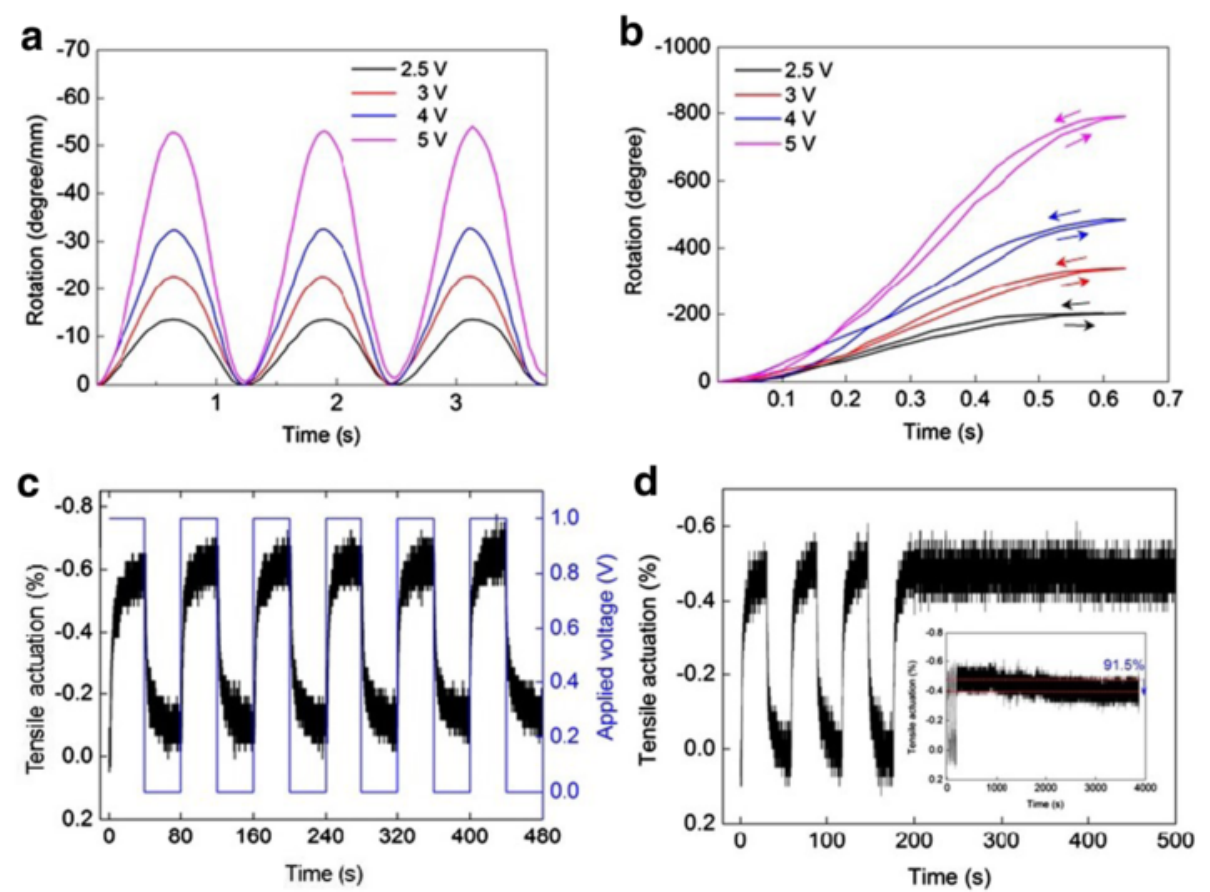

Figure 4 Torsional and tensile actuation for all-solid-state electrochemical CNT muscles. (a) Torsional rotation per muscle length, in response to different applied square-wave voltages, which were measured by paddle rotation for a two-ply, twist-spun, CNT yarn that was impregnated with PVDF-CO-HFP/TEA.BF/PC solid gel electrolyte. The direction of yarn twist was opposite to that applied for yarn plyimg. (b) Data showing the largely non-hysteretic reversal of torsional actuation for the torsional muscle used in (a). (c) Tensile actuation strain and applied voltage versus time for a two-ply yarn muscle, using polyvinyl alcohol/ $\mathrm{H}_{2} \mathrm{SO}_{4}$ electrolyte, while lifting a $11 \mathrm{MPa}$ load. (d) Tensile actuation for the tensile muscle of (c) during three repeated charge ( $1 \mathrm{~V}$ square wave) and discharge cycles that were followed by a hold period, where the power supply was disconnected. Inset: actuator stroke during an extended hold period where no potential was applied, showing that tensile actuation has a latched state, where actuator stroke is largely maintained for an hour without the need for input power [3].

of tensile muscles and the yarn untwist that provides torsional actuation [2]. The main function of the CNT yarn is to confine this actuating yarn guest, insure mechanical durability, enable electrical heating, and provide a helical geometry that can enable torsional actuation and amplify the effect of volume change on tensile actuator stroke.

The methods for making the twisted and coiled CNT yarns are like those above described for the electrochemical methods, and various methods can be used for incorporating high volume fractions of guest in these yarns. These methods include, for example, melt or solution infiltration (which can be followed by in situ polymerization) and biscrolling, in which the guest is deposited on a CNT sheet before twist insertion [2]. Use of paraffin wax as guest provides large yarn volume changes, and thereby large torsional and tensile strokes, so the experimental results described herein use this guest for hybrid yarn muscles.

As is true, for the twist-spun electrochemical muscles, yarn muscle structure and the type of end tethering is key for enabling large muscle strokes (tensile and torsional), as well as long muscle cycle lifetimes. One hundred tensile actuation cycles during lifting have been demonstrated for electrochemically powered a plied, coiled CNT yarn muscle (Figure 5). The tensile actuation did not significantly change during cycles. The chirality of yarn twist versus that of yarn plying is important for determining muscle performance, as is the relative chirality of possibly different segments in a yarn muscle. As a more precise refinement generalization of previous nomenclature [2], we here define a muscle yarn segment as homochiral or heterochiral according to whether or not this segment has the same or different chiralities for yarn twist and yarn plying in this segment.

Figure 6 shows the different muscle structures and configurations that have experimentally investigated for these hybrid yarns [2]. Using opposite chirality plied yarn segments (which are each homochiral), with a paddle at their interconnection (Figure 6d), maximizes initial torque on the paddle, because these segments operate additively to generate rotation. For the same twoply homochiral yarn, but without segmentation into sections having opposite chirality, the one-end-tethered 

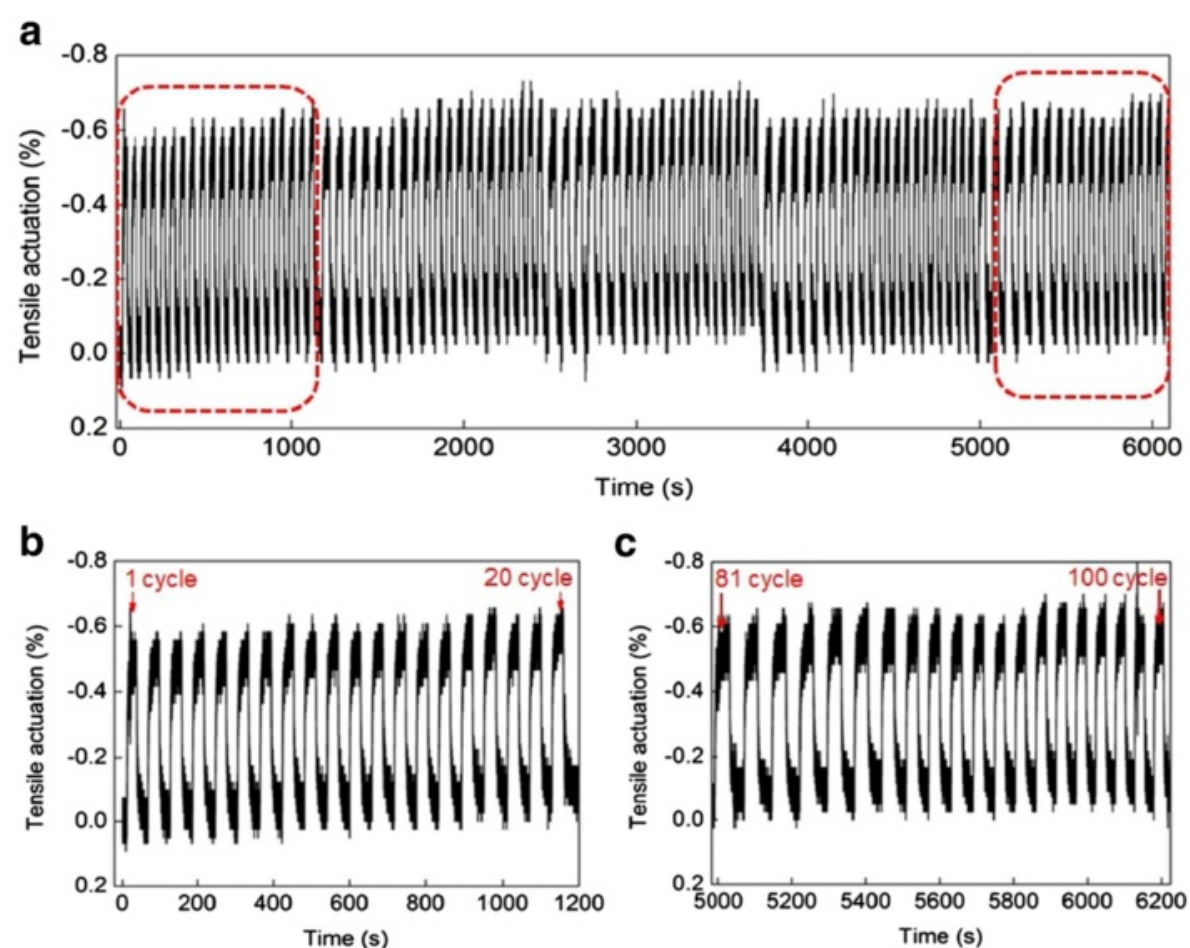

Figure 5 Cycling stability of electricochemically driven tensile actuator in solid electrolyte. (a) Tensile actuation versus time for 100 actuation cycles of the muscle of Figure $3 \mathrm{~h}$. $(\mathbf{b}, \mathbf{c})$ The first and last 20 actuation cycles of (a) using an expanded time scale.

configuration of Figure $6 c$ provides twice the torsional rotation of the Figure $6 \mathrm{~d}$ configuration, but one-half the initial torque, so both configurations provide equal torsional work capacity. Actuation of one segment in a two-end-tethered yarn (Figure $6 \mathrm{~b}$ ) generates smaller paddle rotation than for the same yarn in the configuration of Figure 6d (where two opposite chirality segments are used and both are actuated) because of the energetic cost of twisting the unactuated yarn as the actuating yarn untwists. As for the Figure 6c configuration, the Figure $6 \mathrm{~d}$ configuration with non-plied yarn does not provide reversible actuation unless internally constrained by a solid guest, to prevent left-handed twist ( $\mathrm{Z}$ twist) in one muscle segment from canceling righthanded twist (S twist) in the other yarn segment. Since untwist of the actuating segment is compensated by uptwist of the non-actuated segment in Figure 6a, this actuator configuration can optimize tensile contraction per actuated muscle length. Since the entire yarn untwists during actuation for the Figure $6 \mathrm{c}$ and d configurations (and this untwist lengthens the muscle), they do not provide maximized percent tensile contraction.

Although other guest materials can be used to obtain high performance tensile and torsional hybrid yarn muscles (some of which are more suitable for use in extremely low and high temperature ranges), the presently described results are for prototypical hybrid muscles that use paraffin wax guest. Figure 7 a shows the dramatic increase in tensile actuator stroke that results from the incorporation of paraffin wax in a twist-spun, coiled CNT yarn and the figure inset shows the much smaller thermo-dimensional changes that occur for twisted, non-coiled yarns that either contain wax or are free of guest. Figure $7 \mathrm{~b}$ shows that yarn twist insertion to produce complete coiling results in a large tensile actuator contraction when the neat yarn is heated to extreme temperatures: a $7.3 \%$ reversible tensile contraction upon heating to $\sim 2560^{\circ} \mathrm{C}$ under $3.8-\mathrm{MPa}$ tensile stress, corresponding to $0.16 \mathrm{~kJ} / \mathrm{kg}$ of contractile work capability per yarn weight. The stroke for highly coiled, wax-filled yarn decreases at low applied stresses (Figure 7c), which is consistent with the close proximity of adjacent coils hindering contraction. This figure shows that there is an optimal amount of coiling that maximizes either stroke or work during contraction for the wax hybrid yarn. A maximum contraction of $5.6 \%$ was observed at $5.7 \mathrm{MPa}$ stress for a coiled $\mathrm{CNT}$ yarn having intermediate twist. Adding $6.8 \%$ more twist to the coiled yarn increased the stress of maximum contraction (16.4 $\mathrm{MPa}$ for $5.1 \%$ strain) and the maximum measured contractile work $(1.36 \mathrm{~kJ} / \mathrm{kg}$ for 84 MPa load). 


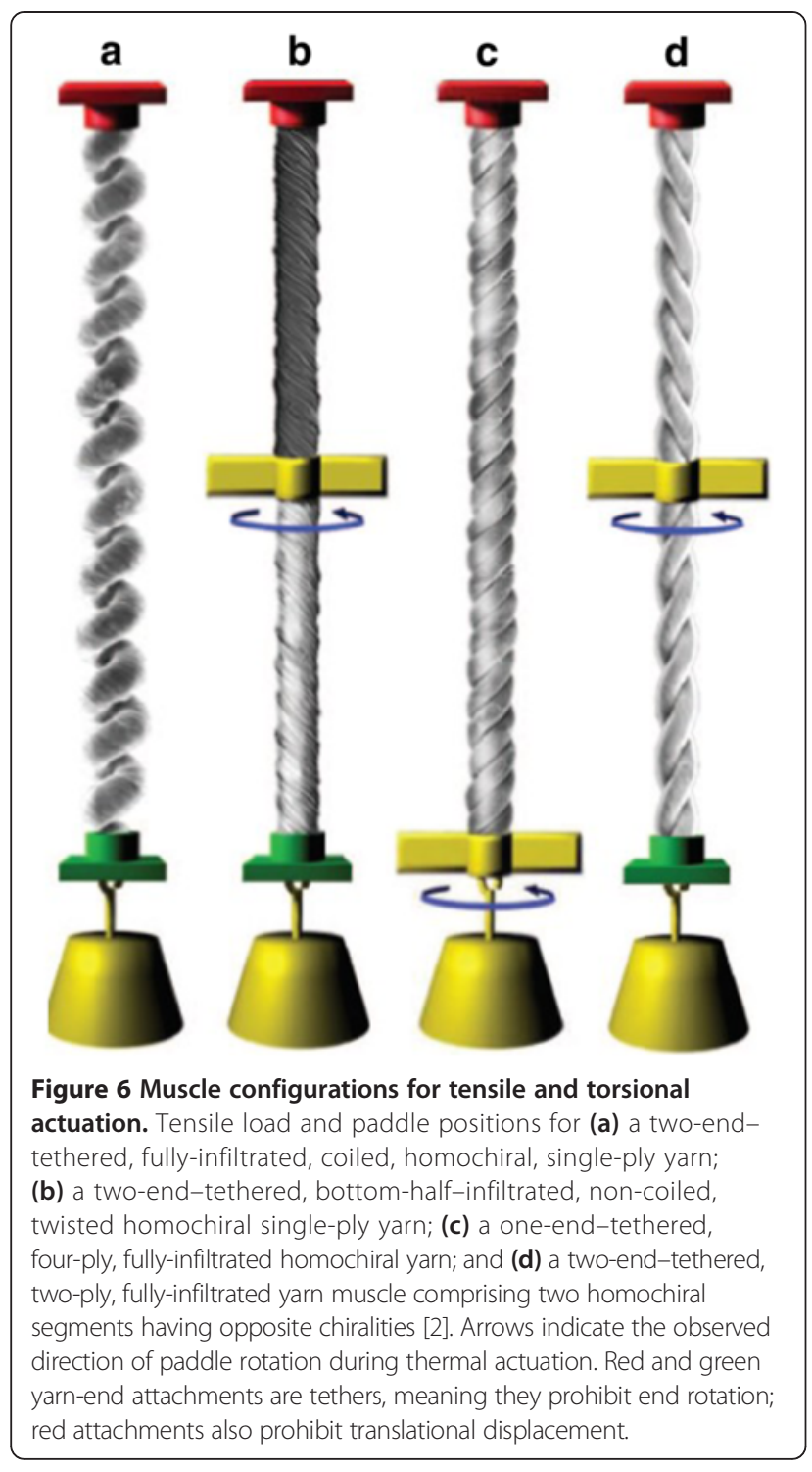

Fast, highly reversible torsional actuation was demonstrated for 2 million cycles for two-end-tethered, halfwax-infiltrated homochiral yarn that rotated a paddle at yarn midpoint (Figure 6b configuration). The hybrid yarn accelerated a 16.5 times-heavier paddle to a fullcycle-averaged 11,500 rotations per minute-first in one direction and then in reverse (Figure 8a). Figure 8b shows the dependence of torsional rotation on input electrical power and applied tensile load for a similar yarn that rotated a 150-times-heavier paddle for a million highly reversible cycles. The average speed was 7,600 revolutions/minute.

\section{Conclusions}

The reviewed work on twist-spun CNT artificial muscles demonstrates the extremely high torsional and tensile muscle performance than can be obtained by the use of twisted, non-coiled and twisted, coiled CNT yarns. The liquid-electrolyte-based twisted, non-coiled muscles (1) generate highly reversible torsional rotation $\left(250^{\circ} / \mathrm{mm}\right.$ of muscle length), which is 1000 times that for prior-art muscles, as well as a 590 revolutions/minute maximum rotation rate that was maintained for 30 full rotations and (2) a gravimetric peak torsional power output similar to that of large, high-power electric motor [1]. While the demonstrated use of these muscles for microfluidic circuits might become practical, the need for an electrolyte bath and associated confinement system is problematic (especially since it dramatically increases system weight and volume).

To eliminate this problem, our team has further demonstrated thermally powered CNT hybrid muscles and all-solid-state electrochemical CNT muscles that can be operated in air $[2,3]$. Paraffin-wax-filled thermally powered CNT hybrid muscles provides 2 million cycles of torsional actuation without evidence of performance decrease with cycling and an average torsional rotation speed of $11,500 \mathrm{rpm}$ (20 times that of our electrochemical torsional muscles). When configured as coiled tensile muscles, these wax-hybrid muscles generated $27.8 \mathrm{~kW} / \mathrm{kg}$ of power during muscle contraction, which is 80 times that for natural skeletal muscles and 30 times that we realized for our electrochemical, liquid-electrolyte CNT muscles.

We next invented all-solid-state electrochemical muscles that avoid the problems of our liquid electrolyte electrochemical muscles - since no liquid bath and associated confinement system is needed, the actuator volume and weight is only that of coupled anode and cathode muscle fibers and associated relatively minor amount of gel electrolyte [3]. The presently realized torsional muscle stroke for plied electrochemical CNT anode and cathode $\left(53^{\circ} / \mathrm{mm}\right.$ of muscle length) is giant compared with muscles other than described here, but still smaller than the $250^{\circ} / \mathrm{mm}$ of muscle length that we obtained for liquidelectrolyte-based muscles. Small, but useable, tensile muscle strokes were obtained $(1.3 \%$ at $2.5 \mathrm{~V}$ and $0.52 \%$ at $1 \mathrm{~V}$ ) when lifting loads that are $\sim 25$ times heavier than can be lifted by the same diameter human skeletal muscle. Very importantly, in contrast to the case for thermal muscles, we demonstrated the existence of a natural latched state, where stroke (contraction) can be largely maintained with little decrease for an hour when the power supply is disconnected.

There are many opportunities for further improvements in these described actuator technologies, such as in obtaining high electrical-to-mechanical energy efficiencies and large tensile strokes for the all-solid-state electrochemical muscles. More recently realized advances in both areas will be described in the future. 

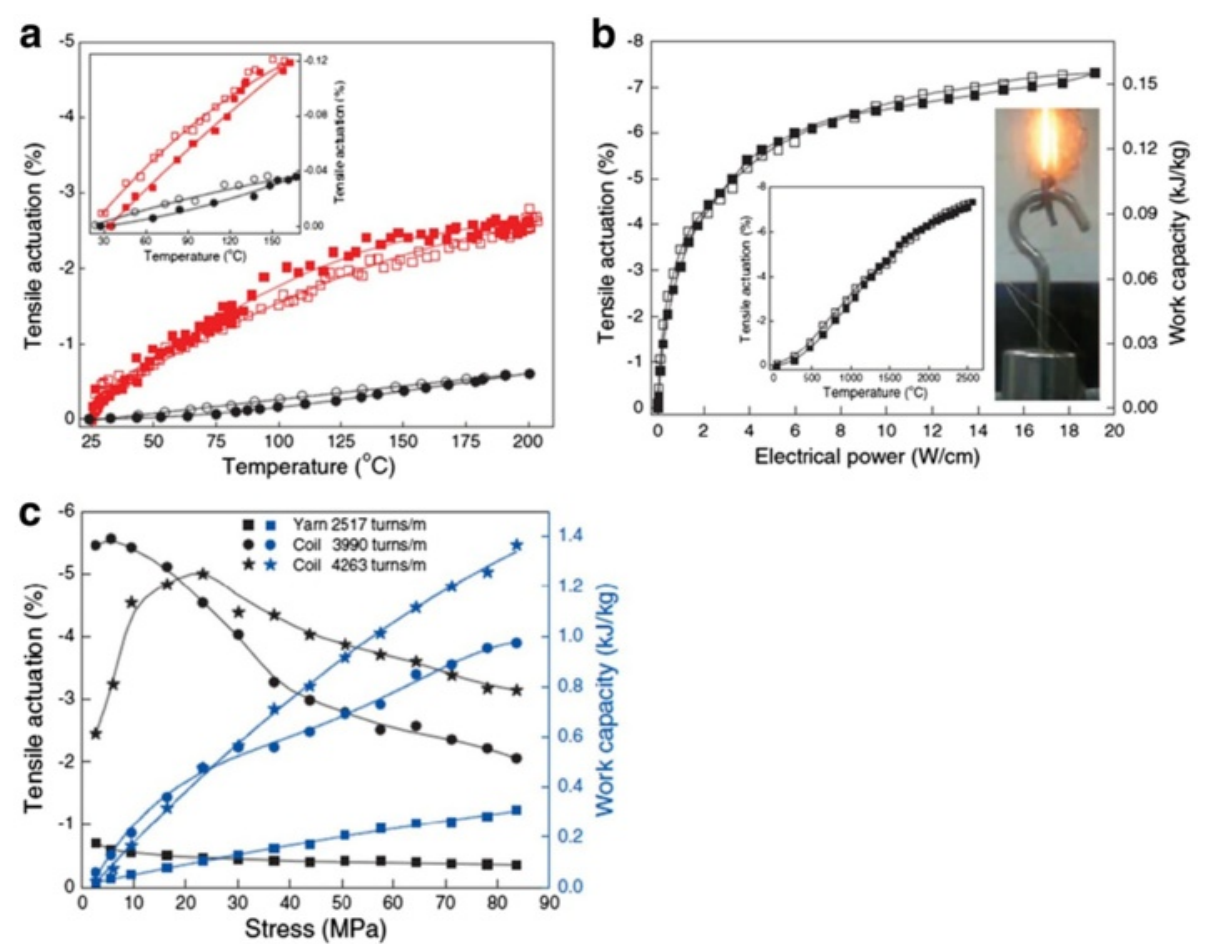

Figure 7 Thermal tensile actuation for two-end-tethered homochiral yarns. (a) Tensile actuation strain versus temperature before (black) and after (red) wax infiltration for a coiled yarn having an applied stress of $6.8 \mathrm{MPa}$. Inset: Corresponding actuation data before (black) and after (red) wax infiltration for a non-coiled yarn having an applied stress of 4.8 MPa. (b) Electrothermal tensile actuation strain and work capacity during contraction in vacuum as a function of applied electrical power for a neat, coiled CNT yarn. Insets: Tensile actuation versus estimated temperature for this neat yarn (left) and photograph of the incandescent yarn lifting a $10 \mathrm{~g}$ load. Closed symbols and open symbols in (a) and (b) are for increasing and decreasing temperature, respectively. (c) The stress dependence of steady-state tensile actuation and contractile work (black and blue data points, respectively) produced by Joule heating $(0.19 \mathrm{~V} / \mathrm{cm})$ for a 150-mm-diameter, coiled yarn having different levels of inserted twist [2].
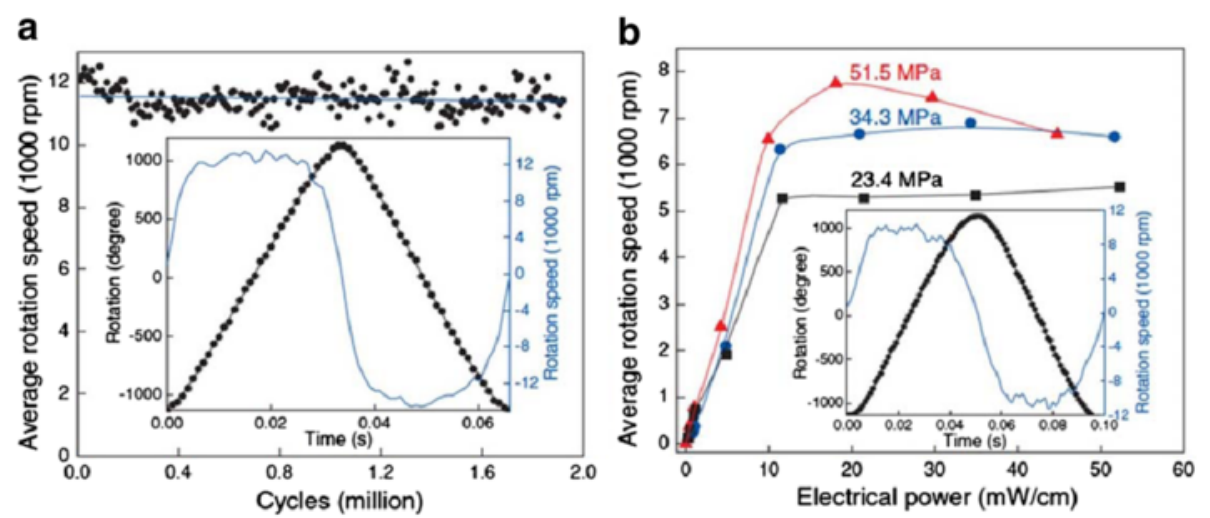

Figure 8 Torsional actuation for two-end-tethered, wax-infiltrated yarns. (a) Average rotation rate versus cycle number when excited by a $15 \mathrm{~Hz}, 40 \mathrm{~V} / \mathrm{cm}$, square-wave voltage using 50\% duty cycle and $41 \mathrm{MPa}$ applied load. Each point on the graph is the average speed over 120 cycles. Inset: rotation angle and rotation speed versus time for one complete cycle (b) Average rotation rate versus applied electrical power for different tensile loads when using the yarn of (a) and a heavier paddle. Inset: rotation angle and speed versus time for 51.5 MPa load [2]. 


\section{Competing interests}

The authors declare that they have no competing interests.

\section{Authors' contributions}

All are participated in the preparation of preparation of manuscript and discussion. All authors read and approved final manuscript.

\section{Acknowledgments}

This work was supported in Korea by the Creative Research Initiative Center for Bio-Artificial Muscle of the Ministry of Science, ICT \& Future Planning (MSIP), the MSIP-US Air Force Cooperation Program (NRF-

2013K1A3A1A32035592) and the Industrial Strategic Technology Program (10038599) and in the United States by Air Force Grant AOARD-13-4119, Air Force Office of Scientific Research grant FA9550-12-1-0211, and Robert A. Welch Foundation grant AT-0029.

\section{Author details}

${ }^{1}$ Center for Bio-Artificial Muscle and Department of Biomedical Engineering, Hanyang University, Seoul 133-791, South Korea. ${ }^{2}$ The Alan G. MacDiarmid NanoTech Institute, University of Texas at Dallas, Richardson, TX 75083, USA.

\section{Received: 14 October 2014 Accepted: 28 October 2014}

\section{Published online: 15 April 2015}

\section{References}

1. J. Foroughi, G.M. Spinks, G.G. Wallace, J. Oh, M.E. Kozlov, S. Fang, T. Mirfakhrai, J.D.W. Madden, M.K. Shin, S.J. Kim, R.H. Baughman, Torsional carbon nanotube artificial muscles. Science 334, 494-497 (2011). doi:10.1126/science.1211220

2. M.D. Lima, N. Li, M.J.D. Andrade, S. Fang, J. Oh, G.M. Spinks, M.E. Kozlov, C.S Haines, D. Suh, J. Foroughi, S.J. Kim, Y. Chen, T. Ware, M.K. Shin, L.D. Machado, A.F. Fonseca, J.D.W. Madden, W.E. Voit, D.S. Galvão, R.H. Baughman, Electrically, chemically, and photonically powered torsional and tensile actuation of hybrid carbon nanotube yarn muscles. Science 338, 928-932 (2012). 10.1126/science.1226762

3. J.A. Lee, Y.T. Kim, G.M. Spinks, D. Suh, X. Lepró, M.D. Lima, R.H. Baughman, S. J. Kim, All solid state carbon nanotube torsional and tensile artificial muscles. Nano Lett 14, 2664-2669 (2014). 10.1021/nl500526r

4. J.A. Lee, M.K. Shin, S.H. Kim, S.J. Kim, G.M. Spinks, G.G. Wallace, R. Ovalle-Robles, M.D. Lima, M.E. Kozlov, R.H. Baughman, Hybrid nanomembranes for high power and high energy density supercapacitors and their yarn application. ACS Nano 6, 327-334 (2012). doi:10.1021/nn203640a

5. J.A. Lee, M.K. Shin, S.H. Kim, H.U. Cho, G.M. Spinks, G.G. Wallace, M.D. Lima, X. Lepro', M.E. Kozlov, R.H. Baughman, S.J. Kim, Ultrafast charge and discharge biscrolled yarn supercapacitors for textiles and microdevices. Nat Commun 4, 1970 (2013). doi: 10.1038/ncomms2970

6. J. Velten, Z. Kuanyshbekova, O. Goktepe, F. Goktepe, A. Zakhidov, Weavable dye sensitized solar cells exploiting carbon nanotube yarns. Appl Phys Lett 102, 203902-203902-3 (2013). doi:10.1063/1.4807891

7. C.H. Kwon, S.H. Lee, Y.B. Choi, J.A. Lee, S.H. Kim, H.H. Kim, G.M. Spinks, G.G. Wallace, M.D. Lima, M.E. Kozlov, R.H. Baughman, S.J. Kim, High-power biofuel cell textiles from woven biscrolled carbon nanotube yarns. Nat Commun 5, 3928 (2014). 10.1038/ncomms4928

8. A.E. Aliev, M.D. Lima, S. Fang, R.H. Baughman, Underwater sound generation using carbon nanotube projectors. Nano Lett 10, 2374-2380 (2010). $10.1021 / \mathrm{nl} 100235 \mathrm{n}$

9. M. Zhang, S. Fang, A.A. Zakhidov, S.B. Lee, A.E. Aliev, C.D. Williams, K.R. Atkinson, R.H. Baughman, Strong, transparent, multifunctional, carbon nanotube sheets. Science 309, 1215-1259 (2005). doi:10.1126/ science.1115311

10. M.D. Lima, S. Fang, X. Lepró, C. Lewis, R. Ovalle-Robles, J. Carretero-González, E. Castillo-Martínez, M.E. Kozlov, J. Oh, N. Rawat, C.S. Haines, M.H. Haque, V. Aare, S. Stoughton, A.A. Zakhidov, R.H. Baughman, Biscrolling nanotube sheets and functional guests into yarns. Science 331, 51-55 (2011). 10.1126/ science.1195912

11. D. Li, W.F. Paxton, R.H. Baughman, T.J. Huang, J.F. Stoddart, P.S. Weiss, Molecular, supramolecular, and macromolecular motors and artificial muscles. MRS Bull 34, 671 (2009). http://dx.doi.org/10.1557/mrs2009.179

12. A.E. Aliev, J. Oh, M. Kozlov, A.A. Kuznetsov, S. Fang, A.F. Fonseca, Giant-stroke, superelastic carbon nanotube aerogel muscles. Science 323, 1575-1578 (2009). 10.1126/science. 1168312
13. R.H. Baughman, C. Cui, A.A. Zakhidov, Z. lqbal, J.N. Barisci, G.M. Spinks, G.G. Wallace, A. Mazzoldi, D.D. Rossi, A.G. Rinzler, O. Jaschinski, S. Roth, M. Kertesz, Carbon nanotube actuators. Science 284, 1340-1344 (1999). 10.1126/ science.284.5418.1340

14. A.M. Fennimore, T.D. Yuzvinsky, W.-Q. Han, M.S. Fuhrer, J. Cumings, A. Zettl, Rotational actuators based on carbon nanotubes. Nature 424, 408-410 (2003). 10.1038/nature01823

15. A.C. Keefe, G.P. Carman, Thermo-mechanical characterization of shape memory alloy torque tube actuators. Smart Mater Struct 9, 665 (2000). 10.1088/0964-1726/9/5/311

16. J. Kim, B. Kang, Performance test and improvement of piezoelectric torsional actuator. Smart Mater Struct 10, 750-757 (2001). 10.1088/0964-1726/10/4/320

17. Y. Fang, T.J. Pence, $X$. Tan, Fiber-directed conjugated-polymer torsional actuator: nonlinear elasticity modeling and experimental validation. IEEE/ ASME Trans Mechatron 16, 656-664 (2011). 10.1109/TMECH.2010.2049366

18. M. Ue, A. Murakami, S. Nakamura, A convenient method to estimate ion size for electrolyte materials design. J Electrochem Soc 149, A1385 (2002). $10.1149 / 1.1507593$

\section{Submit your manuscript to a SpringerOpen ${ }^{\odot}$ journal and benefit from:}

- Convenient online submission

- Rigorous peer review

- Immediate publication on acceptance

- Open access: articles freely available online

- High visibility within the field

- Retaining the copyright to your article

Submit your next manuscript at $>$ springeropen.com 